Title:

\title{
Reduced kidney function at presentation in unselected acute emergency medical admissions : incidence, outcome and associated factors.
}

\section{Running Title:}

Renal dysfunction in emergency medical admissions

\section{Authors:}

Elizabeth Yang ${ }^{1 \mathbf{a}^{\dagger}}$, Anil Chalisey ${ }^{1 \dagger}$, Michael E. Reschen ${ }^{1,2+}$, Brian Shine ${ }^{3}$, Daniel S. Lasserson ${ }^{4}$, Christopher A. $\mathrm{O}^{\prime}$ Callaghan ${ }^{1}$

\section{Affiliations}

${ }^{1}$ Nuffield Department of Clinical Medicine, University of Oxford, Henry Wellcome Building, Roosevelt Drive, Oxford, OX3 7BN, UK.

${ }^{2}$ Oxford University Hospitals NHS Foundation Trust, John Radcliffe Hospital, Oxford, OX3 9DU, UK.

${ }^{3}$ Nuffield Department of Clinical Laboratory Sciences, University of Oxford and Department of Clinical Biochemistry, John Radcliffe Hospital, Oxford, OX3 9DU, UK.

${ }^{4}$ Institute of Applied Health Research, College of Medical and Dental Sciences, University of Birmingham, Edgbaston, Birmingham, B15 2TT, UK

\section{Correspondence to:}

Christopher A. O'Callaghan

Nuffield Department of Clinical Medicine, University of Oxford, Henry Wellcome Building, Roosevelt Drive, Oxford, OX3 7BN, UK.

Tel +44 1865 287789;

Email: chris.ocallaghan@ndm.ox.ac.uk

${ }^{\dagger}$ These authors contributed equally to this work and the name order is arbitrary.

- Current address: Basildon University Hospital, Basildon, Essex, SS16 5NL, UK 


\section{Abstract}

We sought to assess the impact of renal impairment on acute medical admissions and to identify potential contributory factors to admissions involving renal impairment at presentation. In a prospective cohort study, $29.5 \%$ of all acute medical emergency admissions had an eGFR $<60 \mathrm{ml} / \mathrm{min} / 1.73 \mathrm{~m}^{2}$ at presentation. Of these, 19.9 $\%$ had definite chronic kidney disease and $8.4 \%$ had definite acute kidney injury. Detailed analysis of a random subset of patients with an eGFR $<60 \mathrm{ml} / \mathrm{min} / 1.73 \mathrm{~m}^{2}$ at presentation demonstrated that the major reasons for admission included falls, dehydration and fluid overload. $46 \%$ were on diuretics and $53 \%$ were on an ACEI or ARB or both. Gastrointestinal disturbance and recent medication changes were common and diuretic use persisted even with diarrhoea or vomiting. 


\section{Introduction}

A low estimated glomerular filtration rate (eGFR) is common among hospitalized patients and may arise from chronic kidney disease (CKD), acute kidney injury (AKI) or a combination of both. CKD is largely classified on the basis of eGFR and albuminuria, with CKD stage G3 or worse diagnosed by a glomerular filtration rate $<60$ $\mathrm{ml} / \mathrm{min} / 1.73 \mathrm{~m}^{2}$ on at least two occasions over 3 or more months apart ${ }^{1,2}$. CKD is common ${ }^{3}$. In the United Kingdom, the age standardized prevalence of stage 3-5 CKD has been reported as $8.5 \%$, rising substantially with age ${ }^{4}$ and within Oxfordshire, we found the overall unadjusted prevalence to be $4.2 \%{ }^{5}$. The true prevalence may be higher, as CKD is often asymptomatic and many people are untested ${ }^{4,6}$. Kidney function deteriorates with age, so with an ageing population, the prevalence of CKD will increase further ${ }^{7}$. CKD is a major independent risk factor for cardiovascular morbidity, mortality and for hospitalization 8,9 . CKD influences prescribing because renal dysfunction can alter drug pharmacokinetics and because some drugs may worsen renal function ${ }^{10}$. Impaired renal function is itself a risk factor for hospital admissions associated with adverse drug reactions ${ }^{11,12}$.

As with CKD, AKI is also an important predictor of in-hospital morbidity and mortality and the incidence of AKI in hospitalized patients is between 5 and $7 \%{ }^{8,13,14}$, with some studies reporting mortality rates of up to $33 \%$ in these patients ${ }^{14,15}$. The recently published RISK study of a 24 hour snapshot across a set of UK centres showed that $75 \%$

of AKI detected in acute hospital admissions over the next 7 days was acquired in the community ${ }^{16}$. However, both AKI and CKD are of importance in acute illness and in this study, we assessed the prevalence of impaired renal function, due to AKI, CKD or both, in patients admitted to hospital as acute medical emergencies. We then assessed the relationship of kidney disease to length of hospital stay and mortality. We undertook detailed interviews with a random subset of these admitted patients with impaired renal function to identify the factors and community processes of care that contributed to their hospital admission and to identify potential avoidable factors.

\section{Methods}

A prospective cohort study was undertaken of all patients admitted via the acute medical service over a consecutive 40-day period from March to May 2012 in the Oxford University Hospitals, a 1500-bedded acute trust in Oxfordshire, UK, providing both general and specialist care. Patients under 18 years and patients who were already on renal replacement therapy (dialysis or transplantation) prior to admission were excluded from analysis. Patients admitted more than once during the study period were included only on their first admission. The final study group comprised 2070 patients. eGFR was calculated using the Modification of Diet in Renal Disease study (MDRD) formula as this was in clinical use in the hospital ${ }^{17}$. Our analysis plan focused on the cohort of patients with an eGFR $<60 \mathrm{ml} / \mathrm{min} / 1.73 \mathrm{~m}^{2}$ at presentation. These patients were classified as having AKI in accordance with the KDIGO/AKI network criteria if they had a rise in serum creatinine of $\geq 26.5 \mu \mathrm{mol} / \mathrm{l}$ or an increase of $\geq 1.5$ fold. If there was a rise in serum creatinine of $\geq 26.5 \mu \mathrm{mol} / \mathrm{l}$ from a pre-existing (within preceding 12 months) eGFR baseline value of $<60 \mathrm{ml} / \mathrm{min} / 1.73 \mathrm{~m}^{2}$, the patient was classified as having AKI-on-CKD. Patients with a persistent eGFR $<60 \mathrm{ml} / \mathrm{min} / 1.73 \mathrm{~m}^{2}$ and creatinine change of $<26.5 \mu \mathrm{mol} / \mathrm{l}$ were classified as having CKD. In certain cases, where renal function data for comparison were unavailable, the patient was deemed to be unclassifiable. A random (coin toss) sample of 152 patients with an eGFR $<60 \mathrm{ml} / \mathrm{min} / 1.73 \mathrm{~m}^{2}$ were analyzed in more detail by interview and medical record review in order to determine medical and drug history and factors 
leading to admission. Participants provided informed consent and ethical approval for this study was granted by the UK National Research Ethics Service committee East Midlands - Nottingham 1. The presenting syndrome recorded was based on that of the attending clinicians. Statistical analysis was undertaken using $\mathrm{R}^{18}$.

\section{Results}

\section{Renal profile of all acute admissions}

Of the 2070 patients admitted through the acute medical service during the study period 967 (46.7\%) were male. The overall mean age was $64.1 \pm 21.6$ (mean $\pm \mathrm{SD}$ ) and the mean admission eGFR was $75.3 \pm 34.5 \mathrm{ml} / \mathrm{min} / 1.73 \mathrm{~m}^{2}$ (mean \pm SD). Overall, 611 (29.5\%) of the 2070 admitted patients had an eGFR $<60 \mathrm{ml} / \mathrm{min} / 1.73 \mathrm{~m}^{2}$ at presentation and the proportion with this level of renal impairment rose with age (Figure $1 \mathrm{~A}$ and Figure $1 \mathrm{~B}$ ).

Of those patients with renal dysfunction on admission, 412 (19.9\%) had pre-existing renal disease with CKD stage 3 or worse (Figure 1). Of these 412 patients, 112 had AKI-on-CKD and the remainder had stable CKD. 199 patients presented with newly diagnosed renal impairment; of these, 71 were unclassifiable as no previous creatinine was available for comparison. Of the remaining 128 patients with newly diagnosed renal impairment, 61 met the criteria for AKI. In total, 173 (8.4\%) patients met the criteria for AKI on admission of whom 112 patients had AKIon-CKD and 61 had AKI alone.

Following discharge, a total of 154 patients with an admission eGFR of $<60 \mathrm{ml} / \mathrm{min} / 1.73 \mathrm{~m}^{2}$ showed recovery of renal function with an eGFR $>60 \mathrm{ml} / \mathrm{min} / 1.73 \mathrm{~m}^{2} .40(65.6 \%)$ of the patients with only AKI, $27(40.3 \%)$ of the patients with new renal impairment who did not fulfil the criteria for AKI and 21 (29.6\%) of the patients who were 'unclassifiable' recovered renal function on discharge. In contrast, only 26 (8.6\%) of patients with stable CKD had an eGFR $>60 \mathrm{ml} / \mathrm{min} / 1.73 \mathrm{~m}^{2}$ following discharge.
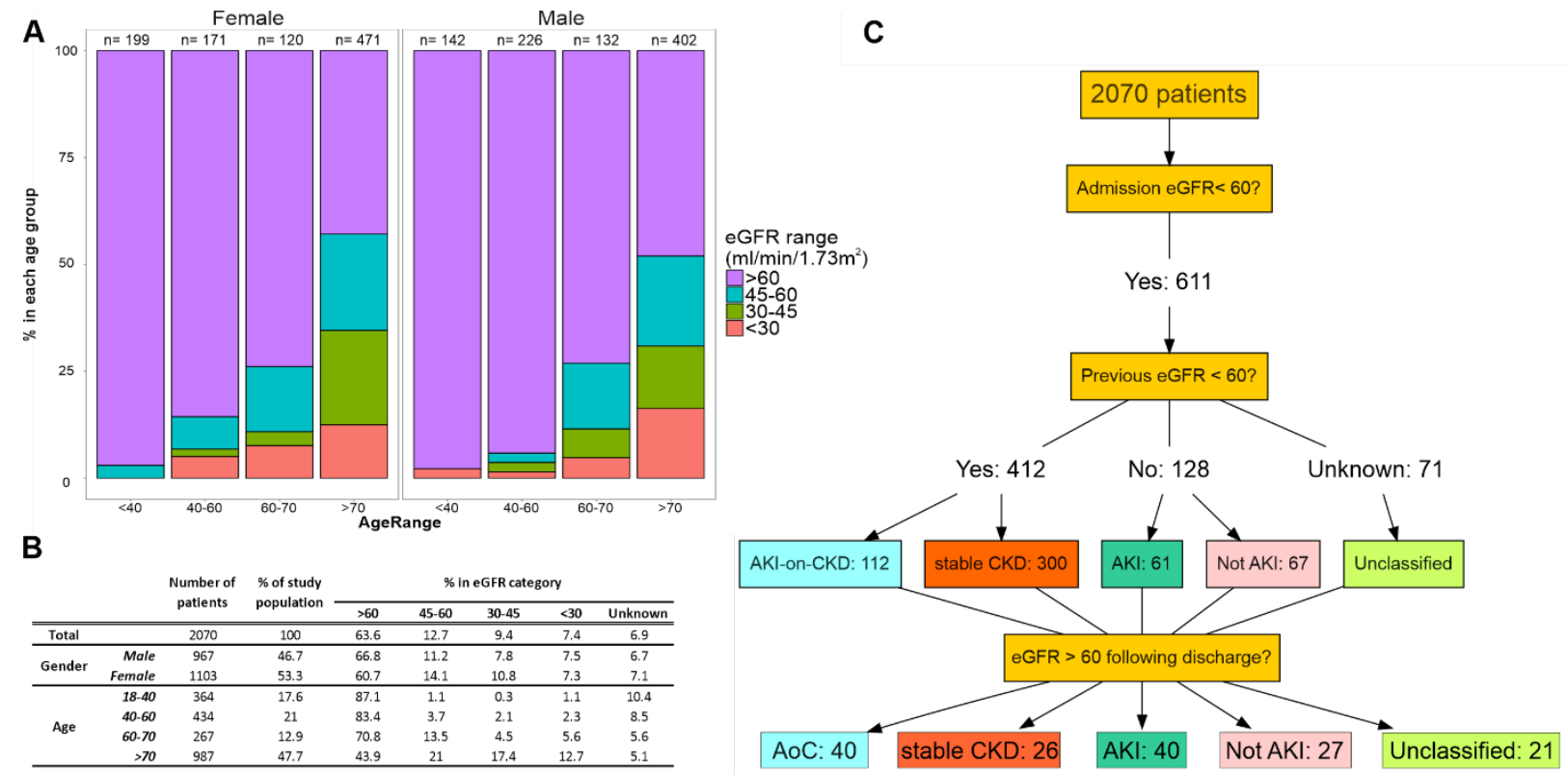

B

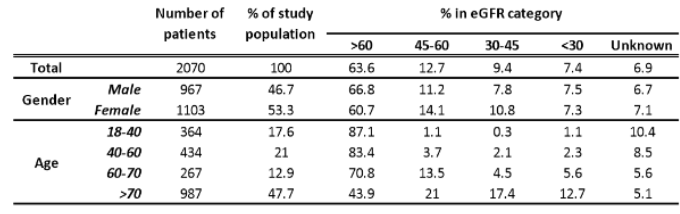


Figure 1: A: Renal profile of patients at the point of admission to hospital by age and gender. The colors indicate eGFR in $\mathrm{ml} / \mathrm{min} / 1.73 \mathrm{~m}^{2}$. Patients for whom it was not possible to calculate eGFR at admission have not been included in this figure. B: Renal profile of patients at the point of admission to hospital by age and gender. C: Admission status, progress and renal outcome of patients admitted to the acute medical service during the study period.

\section{Detailed analysis of pre-admission factors}

A randomly selected set of 152 patients with an admission eGFR of $<60 \mathrm{ml} / \mathrm{min} / 1.73 \mathrm{~m}^{2}$ were analyzed in detail by review of their healthcare records and interviews with the patients and clinicians. The following results relate to this in depth study of these patients, whose demographic and clinical characteristics are shown in Figure 2; overall, patients in this population with renal impairment were relatively elderly with a high burden of cardiovascular disease. They presented with substantial renal impairment with 31 patients having a creatinine > $200 \mu \mathrm{mol} / \mathrm{l}$ and 45 having an eGFR $<30 \mathrm{ml} / \mathrm{min} / 1.73 \mathrm{~m}^{2}$.

A

\begin{tabular}{lrr}
\hline Gender & & \\
\hline Male & 75 & $49.3 \%$ \\
Female & 77 & $50.7 \%$ \\
\hline & Mean age & Median age (range) \\
\hline Male & 79.6 & $82(40-97)$ \\
Female & 81 & $83(47-98)$ \\
\hline Ethnicity & & \\
\hline Caucasian & 147 & $96.7 \%$ \\
Black & 2 & $1.3 \%$ \\
Asian & 1 & $0.7 \%$ \\
Other & 1 & $0.7 \%$ \\
\hline Hypertension & 8 & $5.3 \%$ \\
\hline Pre-existing vascular disease & \\
\hline CAD & 34 & $22.4 \%$ \\
TIA/Stroke & 26 & $17.1 \%$ \\
PVD & 11 & $7.2 \%$ \\
\hline Smoker & & \\
\hline Ex & 65 & $42.8 \%$ \\
Current & 14 & $9.2 \%$ \\
\hline Diabetes & 1 & $0.7 \%$ \\
\hline & &
\end{tabular}

B

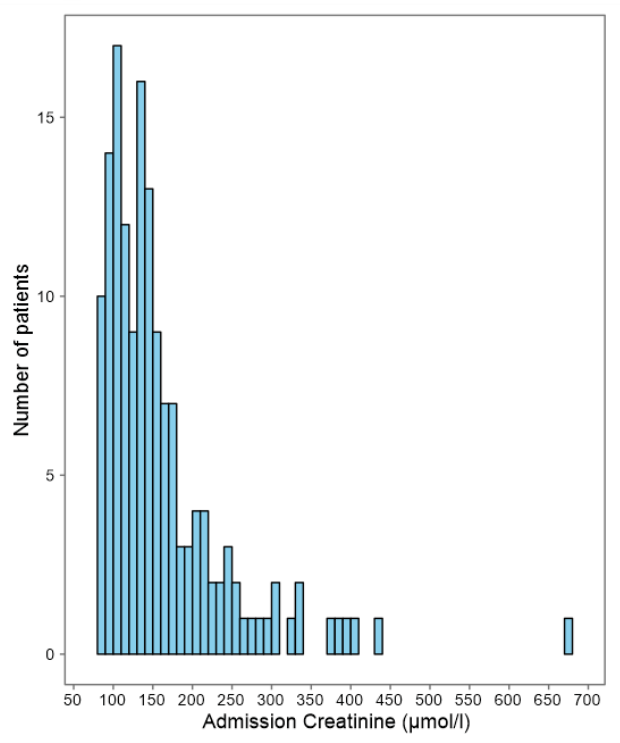

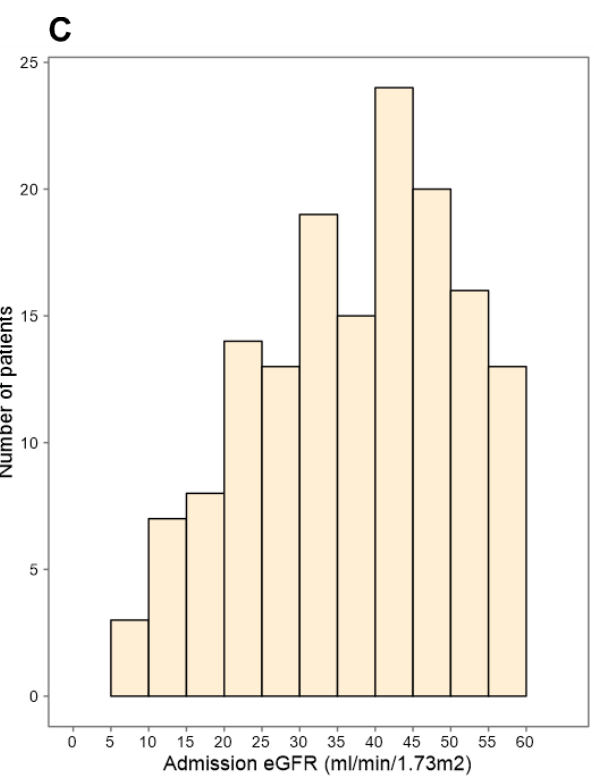

Figure 2: A: Characteristics of the subset of patients who were studied in detail. CAD, coronary artery disease; TIA, transient ischemic attack; PVD, peripheral vascular disease. B: Histogram of creatinine values on admission. C: Histogram of eGFRs on admission.

\section{Reason for admission}

The principal reason for admission in this in depth analysis was determined by the admitting clinician and generally fell into a relatively small set of categories, with falls, disturbance in fluid balance and infections predominating (Figure 3). A broad range of eGFRs was associated with each admission category. A Kruskall-Wallis test showed a 
significant difference in eGFR between admission categories $(p<0.0005)$. A pairwise comparison was performed between all groups using the Dunn test, revealing significant differences between the 'intravascularly depleted' category compared to 'falls', 'other' and 'collapse' ( $p=0.002,0.003$, and 0.007 , respectively) and between the 'urinary tract infection' and 'other' category $(p=0.04)$.

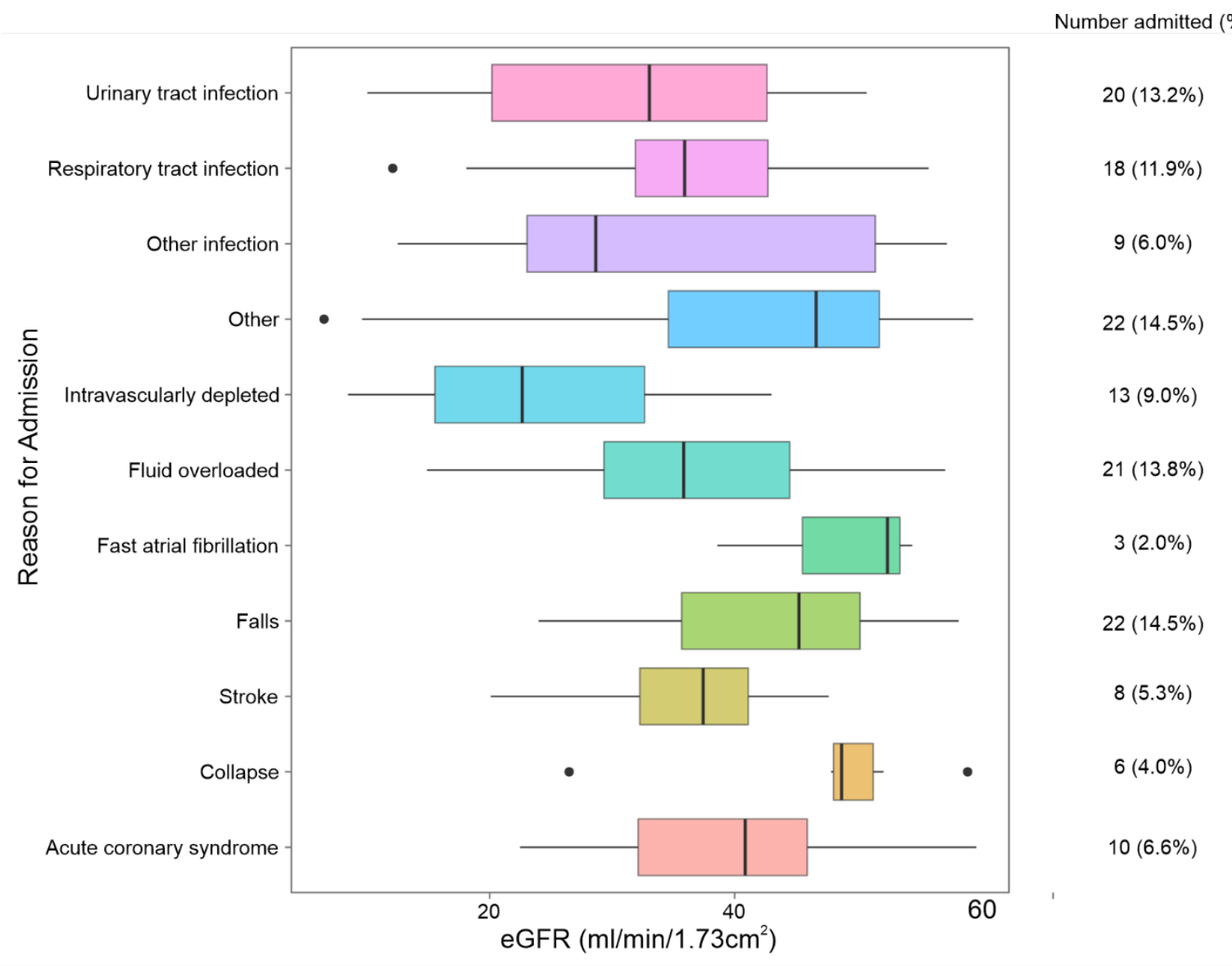

Figure 3: Boxplot illustrating the eGFR at admission according to reason for admission. The figures to the side show the number and percentage of patients in each category. Thick vertical lines represent the median value, and rectangles contain the interquartile range. Horizontal lines represent the range from the minimum to maximum values, excluding outliers, which are shown as dots.

In $34(22 \%)$ of the 152 patients studied in detail, the principal reason for admission was an abnormality in fluid volume status. Regardless of the principal reason for admission, 76 (50\%) of all patients studied were judged by their clinicians to have had abnormal fluid balance of which 47 (31\%) had intravascular fluid depletion and 29 (19\%) had fluid overload. Frank peripheral oedema was noted in 45 (29.6\%) of patients, including 4 of the 13 patients admitted with a primary reason for admission of intravascular depletion and 6 of the 22 patients admitted following falls.

Fluid depletion and medication 
Intravascular fluid depletion was a common reason for admission and was associated with the lowest mean eGFR (Figure 3). Gastrointestinal disturbance was common across all patients interviewed, with 77 (51\%) complaining of vomiting and/or diarrhoea before admission (Figure 4). In particular, 11 (85\%) of 13 patients with intravascular depletion as the principal reason for admission had vomited or had diarrhoea before admission. Of all 77 patients with diarrhoea and/or vomiting, 56 were on a diuretic or a renin-angiotensin-aldosterone (RAAS) blocker (an angiotensin converting enzyme inhibitor (ACEi) or an angiotensin receptor blocker (ARB)) and none had stopped taking their medication.

Overall, 70 (46\%) of all patients studied were on diuretics, 58 (39\%) were on an ACEi and 22 (14\%) on an ARB (Figure 4A). 41 (27\%) of patients were taking both a diuretic and an ACEI or ARB. Only 44 (29\%) of patients were not on either a diuretic, or RAAS blockade. Of the 70 taking a diuretic, 52 (74\%) were taking furosemide, 13 (18\%) were taking bendroflumethiazide and $5(7 \%)$ were taking spironolactone. 12 out of the 13 patients whose principal reason for admission was intravascular fluid depletion were on either a diuretic or an RAAS blocker and 3 of these patients were on both. Only 2 of the patients with falls were not on a diuretic, a RAAS blocker or both.

\section{Fluid overload and medication}

In the 21 patients with fluid overload as the reason for admission, 4 (19\%) were not on a diuretic. In addition, 4 (19\%) of those with fluid overload as the principal reason for admission were taking non-steroidal antiinflammatory drugs (NSAIDs) in addition to their diuretic or RAAS blocker. 33 (21\%) of all patients with renal dysfunction at admission were using NSAIDs, and of this subset, 21 (64\%) were also taking a diuretic or RAAS blocker or both.

\section{Medication and renal dysfunction}

The number of prescribed drugs taken by patients was high and only 2 patients were taking no medication (Figure 4). 126 (83\%) were taking 5 or more drugs, 47 (31\%) were taking 10 or more drugs and 14 (9\%) were taking 15 or more drugs. 35 (23\%) of the 152 patients studied had prescriptions that were not optimal for their level of renal function in the light of national guidance in the British National Formulary. The dose of a loop diuretic was often lower than expected for the level of renal function or a thiazide was used when a loop diuretic would have been more appropriate for the patient's level of renal function. Drugs that were prescribed at doses that were too high for the level of renal function included allopurinol, metformin and digoxin. 
A

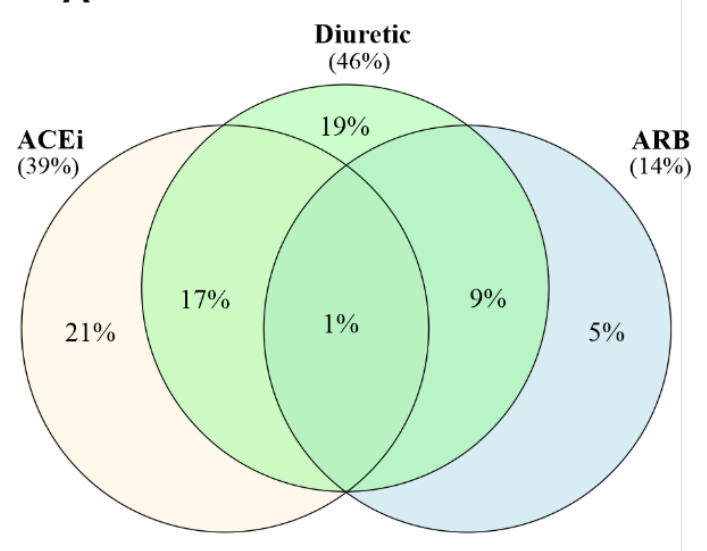

B

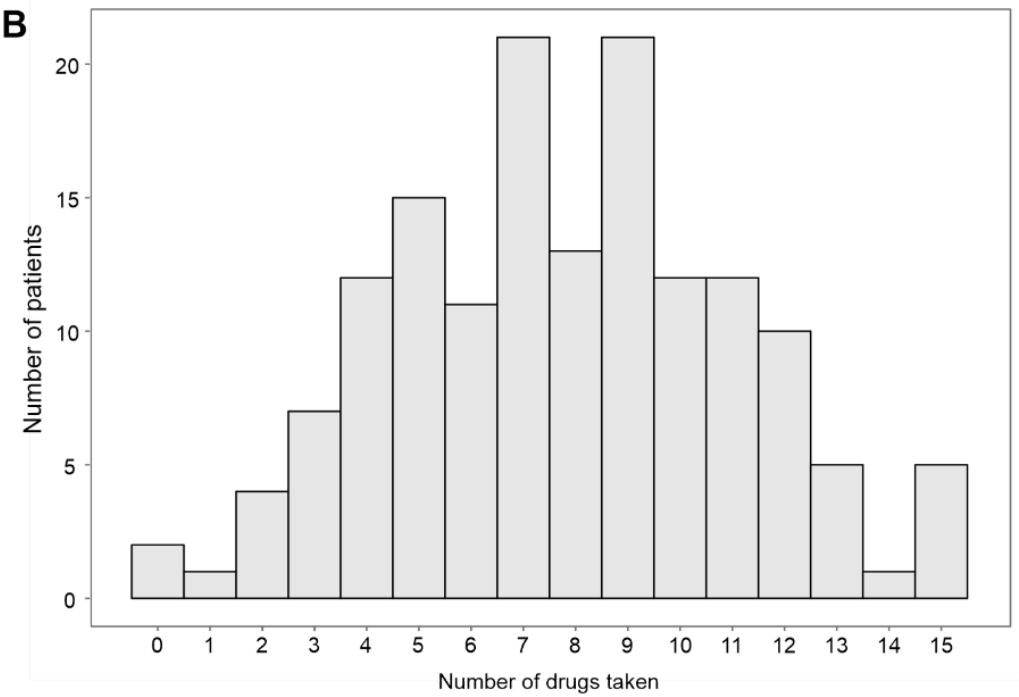

C

\begin{tabular}{r|cccc} 
& Total & On diuretic & $\begin{array}{c}\text { On RAAS } \\
\text { blocker }\end{array}$ & On both \\
\hline Vomiting alone & 21.7 & 5.3 & 4.6 & 4.6 \\
$\begin{array}{r}\text { Diarrhoea alone } \\
\begin{array}{r}\text { Diarrhoea and } \\
\text { vomiting }\end{array}\end{array}$ & 14.5 & 1.3 & 7.2 & 2.6 \\
Total & 14.5 & 3.3 & 4 & 4 \\
50.7 & 9.9 & 15.8 & 11.2
\end{tabular}

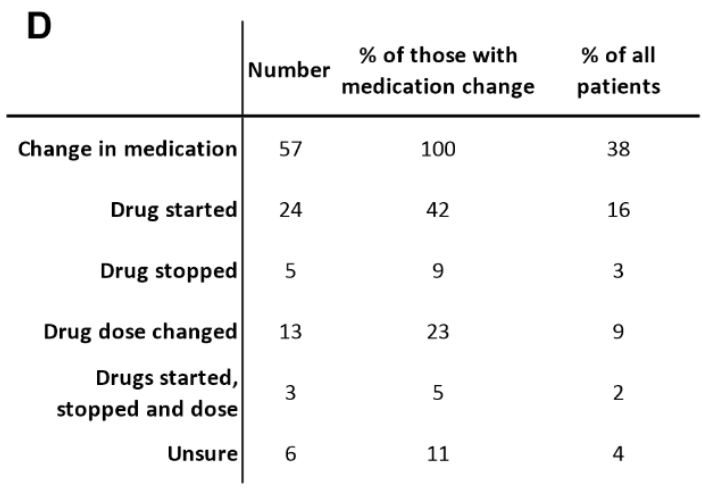

Figure 4: A: Venn diagram of the overlap of prescriptions for the drug classes indicated. Percentages shown are of all 152 patients. B: Histogram showing the distribution of the number of prescribed drugs for each patient. C: Percentages of all 152 patients who had diarrhoea and/or vomiting. D: Changes to prescribed medication before admission.

\section{Pre-admission medication changes}

Overall, 57 (38\%) of patients reported a recent change in their medication, with 30 (20\%) reporting changes within the 14 days preceding admission (Figure 4D). 24 (16\%) of patients had recently started a new drug, 13 (9\%) had a dose change and 5 (3\%) had stopped a drug; other patients were unsure about the nature of the change. A recent change in medication had occurred for $8(38 \%)$ of the patients who had fluid overload as the principal reason for admission, for 4 (31\%) of the patients with intravascular fluid depletion and for $4(18 \%)$ of the patients with falls. Patients with fluid overload who were taking regular diuretics had not increased their diuretic dose despite noticing increased shortness of breath and ankle swelling. In no case had any patient stopped taking their diuretic, RAAS blocker or NSAID when they had vomiting or diarrhoea.

\section{Health understanding}


When interviewed at admission $78(51 \%)$ of patients described their health as average or better and $49(32 \%)$ as worse than average. 14 (9\%) were current smokers and 65 (43\%) were ex-smokers. $84(55 \%)$ recalled being told at some time that they had high blood pressure, but only 39 (26\%) recalled a doctor or nurse discussing their kidneys with them. In only 31 (20\%) did the referral letter, if there was one, mention CKD or renal impairment (Figure 5A).

\begin{tabular}{lrr}
\hline & Number & Percentage \\
\hline Health perception & 5 & 3.29 \\
\hline Very good & 32 & 21.05 \\
Good & 41 & 26.97 \\
Average & 46 & 30.26 \\
Bad & 3 & 1.97 \\
Very bad & & \\
\hline \hline Smoking status & 65 & 42.76 \\
\hline Ex & 58 & 38.16 \\
Never & 14 & 9.21 \\
Current & & \\
\hline \hline Pre-existing knowledge & & \\
of own renal function & 93 & 61.18 \\
\hline No & 39 & 25.66 \\
Yes & & \\
\hline \hline CKD mentioned in & & \\
referral & 85 & 55.92 \\
\hline No & 30 & 19.74 \\
\hline \hline
\end{tabular}

Figure 5: Table of patient reported health status and of referral information..

\section{Discussion}

There is an increasing recognition of the prevalence and significance of chronic and acute kidney disease. The current study was designed to understand the healthcare burden of kidney disease at the front door in acute hospital medicine and to identify potential factors contributing to hospital admissions in people with impaired renal function at the time of their acute hospital presentation. CKD is associated with an increased risk of hospitalization in elderly people in the UK ${ }^{19}$ and in blood samples taken in the community in Oxfordshire we previously reported a prevalence of CKD on the basis of eGFR of around $15 \%{ }^{5}$. AKI is a significant predictor of mortality, length of stay and healthcare cost ${ }^{13,14}$ and has been documented during around $25 \%$ of acute hospital admissions with over half of these patients already having AKI on admission ${ }^{20}$.

A UK study in 2005 found that $19 \%$ of acute medical admissions had an eGFR $<60 \mathrm{ml} / \mathrm{min} / 1.73 \mathrm{~m}^{2}$ at some point during their admission ${ }^{21}$, but the UK population has continued to age with marked increases in the number of older patients seen as acute medical emergencies ${ }^{22}$. An Australian study of admissions in 2007 found an eGFR of $<60 \mathrm{ml} / \mathrm{min} / 1.73 \mathrm{~m}^{2}$ in around $30 \%$ of patients, but did not analyze the cause of these admission or study variables such as fluid status and medication ${ }^{23}$. In the UK most acute patients with a reduced eGFR are not assessed by a specialist renal unit unless there is a specific suspicion of a renal disorder that needs acute treatment or diagnosis 
or the need for renal replacement therapy in the absence of a need for generalized intensive care. We therefore studied the burden of renal impairment in unselected acute general medical presentations.

We focused our study on a point estimate of renal function at the time of admission as this is what the acute physician is commonly presented with and there is good evidence that even a single eGFR $<60 \mathrm{ml} / \mathrm{min} / 1.73 \mathrm{~m}^{2}$ is associated with increased risk of mortality ${ }^{9}$. Overall, we found that nearly one third of patients assessed as medical emergencies had blood results consistent with an acute or chronic reduction in eGFR. The rise in this proportion with age is consistent with the fall in eGFR that occurs with age.

Disturbances of body fluid volume were associated with impaired kidney function at presentation. This may reflect the relative inflexibility of the renal response to change in patients with poor renal function and a drug burden that often includes diuretics, ACEi and ARB, which can further impair this response. With excess gastrointestinal fluid loss from vomiting or diarrhoea, a patient with a diminished eGFR who is taking diuretics is unlikely to conserve fluid as well as someone with good kidney function who is not on a diuretic. Similarly, ACEi or ARB will reduce the effects of angiotensin and aldosterone on the kidney, both of which would normally promote salt and fluid retention in the presence of volume depletion. A patient who is acutely volume depleted does not usually need to continue to take their diuretic. However, we found that patients in this situation did continue to take their diuretics. In this context, we conclude that sick day guidance could help with advice for patients to contact their doctor to discuss whether they should omit their diuretic. With appropriate education, careful self-monitoring of daily weight could empower patients to take decisions about whether to continue their diuretic therapy.

Many patients were taking a large number of medications and the extent to which some, especially the older patients, were able to take more than 10 medications in one day reliably is unclear. A recent change in medication was common and it is unclear whether this was a contributory factor to the presentation or merely a marker for the patient being unwell prior to their hospital presentation and so having their prescription adjusted in the community setting. A detailed study of prescribing for elderly patients with CKD found that $13 \%$ of prescriptions were potentially inappropriate ${ }^{24}$. Impaired renal function was associated with a 2.6 fold increase in the risk of admission due to medication-related problem ${ }^{10}$. We found that the use of NSAIDs was higher than might be anticipated. NSAIDs are not usually prescribed in the context of renal impairment because they can lead to a further reduction in glomerular filtration rate and can promote sodium retention, so antagonizing the effects of diuretics.

There was relatively scant communication about kidney disease between medical teams and few patients recalled discussions with their clinicians about their kidneys. The acute situation presents an opportunity to highlight ways in which patient or their carers might modify their behavior to try to reduce the probability of readmission. A brief conversation about kidney function might be helpful in some cases and further studies are needed to explore this. Simple interventions might include regular home weighing of patients on diuretics and early contact with their general practitioner to discuss what to do about their diuretics when they experience diarrhoea or vomiting. This advice can be usefully consolidated by documenting it in the discharge summary that goes to both the patient and their primary care physician. Thus, there is an important and valuable opportunity, even in the busy and sometimes brief acute setting to make a significant contribution to the long term care of the patient in this way through guidance to both the patient and to their general practitioner. 
Overall, our findings indicate that renal function is impaired in a substantial number of acute medical presentations. This has implications for patients, for healthcare planning and for training in primary and secondary care. It is important for clinicians to recognize the role of renal impairment in acute illness and to prescribe accordingly. With appropriate support patients might be better empowered to manage themselves, especially with respect to diuretics and other medications, to reduce the probability of an acute crisis requiring hospital care. Studies are indicated to establish the extent to which teamwork between patients, primary and secondary care could help to reduce admissions, especially those due to disturbances of body fluid volume regulation.

\section{References}

1. Group., K.D.I.G.O.K.C.W., KDIGO 2012 Clinical Practice Guideline for the Evaluation and Management of Chronic Kidney Disease. Kidney Int Suppl 2013; 3: 1-150.

2. National Kidney, F., K/DOQI clinical practice guidelines for chronic kidney disease: evaluation, classification, and stratification. Am J Kidney Dis 2002; 39: S1-266.

3. Coresh, J., E. Selvin, L.A. Stevens, et al., Prevalence of chronic kidney disease in the United States. JAMA 2007; 298: 2038-47.

4. Stevens, P.E., D.J. O'Donoghue, S. de Lusignan, et al., Chronic kidney disease management in the United Kingdom: NEOERICA project results. Kidney Int 2007; 72: 92-9.

5. O'Callaghan, C.A., B. Shine, and D.S. Lasserson, Chronic kidney disease: a large-scale population-based study of the effects of introducing the CKD-EPI formula for eGFR reporting. BMJ Open 2011; 1: e000308.

6. John, R., M. Webb, A. Young, et al., Unreferred chronic kidney disease: a longitudinal study. Am J Kidney Dis 2004; 43: 825-35.

7. Office for National Statistics. Population. 2013 [cited 2014 October 10, 2014]; Available from:

8. Go, A.S., G.M. Chertow, D. Fan, et al., Chronic kidney disease and the risks of death, cardiovascular events, and hospitalization. N Engl J Med 2004; 351: 1296-305.

9. Weiner, D.E., M. Krassilnikova, H. Tighiouart, et al., CKD classification based on estimated GFR over three years and subsequent cardiac and mortality outcomes: a cohort study. BMC Nephrol 2009; 10: 26.

10. Leendertse, A.J., E.A. van Dijk, P.A. De Smet, et al., Contribution of renal impairment to potentially preventable medication-related hospital admissions. Ann Pharmacother 2012; 46: 625-33.

11. Leendertse, A.J., A.C. Egberts, L.J. Stoker, et al., Frequency of and risk factors for preventable medication-related hospital admissions in the Netherlands. Arch Intern Med 2008; 168: 1890-6.

12. McDonnell, P.J. and M.R. Jacobs, Hospital admissions resulting from preventable adverse drug reactions. Ann Pharmacother 2002; 36: 1331-6.

13. Chertow, G.M., E. Burdick, M. Honour, et al., Acute kidney injury, mortality, length of stay, and costs in hospitalized patients. J Am Soc Nephrol 2005; 16: 3365-70.

14. Xue, J.L., F. Daniels, R.A. Star, et al., Incidence and mortality of acute renal failure in Medicare beneficiaries, 1992 to 2001. J Am Soc Nephrol 2006; 17: 1135-42.

15. Nash, K., A. Hafeez, and S. Hou, Hospital-acquired renal insufficiency. Am J Kidney Dis 2002; 39: 930-6.

16. Risk, l., Risk prediction for acute kidney injury in acute medical admissions in the UK. QJM 2019; 112: 197-205.

17. Levey, A.S., J.P. Bosch, J.B. Lewis, et al., A more accurate method to estimate glomerular filtration rate from serum creatinine: a new prediction equation. Modification of Diet in Renal Disease Study Group. Ann Intern Med 1999; 130: 461-70. 
18. Team, R.C., R: A Language and Environment for Statistical Computing. 2015, R Core Team: Vienna, Austria.

19. Nitsch, D., B.A. Nonyane, L. Smeeth, et al., CKD and hospitalization in the elderly: a community-based cohort study in the United Kingdom. American journal of kidney diseases : the official journal of the National Kidney Foundation 2011; 57: 664-72.

20. Challiner, R., J.P. Ritchie, C. Fullwood, et al., Incidence and consequence of acute kidney injury in unselected emergency admissions to a large acute UK hospital trust. BMC Nephrol 2014; 15: 84.

21. Annear, N.M., D. Banerjee, J. Joseph, et al., Prevalence of chronic kidney disease stages 3-5 among acute medical admissions: another opportunity for screening. QJM 2008; 101: 91-7.

22. Blunt, I., M. Bardsley, and J. Dixon, Trends in Emergency Admissions in England 2004-2009, in New Frontiers in NHS Efficiency. 2010, Nuffield Trust: London.

23. Yong, T.Y., J.S. Fok, P.Z. Ng, et al., The significance of reduced kidney function among hospitalized acute general medical patients. QJM 2013; 106: 59-65.

24. Jones, S.A. and S. Bhandari, The prevalence of potentially inappropriate medication prescribing in elderly patients with chronic kidney disease. Postgrad Med J 2013; 89: 247-50. 